\title{
TIME VARYING ROTATING DISK FLOW WITH HEAT TRANSFER OF A NON-NEWTONIAN FLUID IN POROUS MEDIUM
}

\author{
Hazem Ali Attia ${ }^{1}$, Mostafa A. M. Abdeen ${ }^{2}$ and Menna Mahmoud Elbarawy ${ }^{1}$ \\ ${ }^{1}$ Department of Engineering Mathematics and Physics, Faculty of Engineering, \\ El-Fayoum University, El-Fayoum 63514, Egypt \\ E-mail: ah1113@yahoo.com \\ ${ }^{2}$ Department of Engineering Mathematics and Physics, Faculty of Engineering, Cairo University, \\ Giza 12211, Egypt
}

(Received July 23, 2013)

\begin{abstract}
Time varying flow through a porous medium of an incompressible viscous non-Newtonian fluid due to a rotation of an infinite rotating disk is studied with heat transfer. Numerical solutions using finite differences are obtained for the nonlinear partial differential equations which govern the hydrodynamics and energy transfer. The effect of the porosity of the medium and the characteristics of the non-Newtonian fluid on the velocity and temperature distributions is considered.
\end{abstract}

Key words: Rotating disk flow, non-Newtonian fluid, porous medium, numerical solution, heat transfer.

\section{INTRODUCTION}

The steady flow due to an infinite rotating disk was handled by VON KARMAN in 1921 [1] who gave a formulation for the problem and introduced his famous transformations which reduced the governing partial differential equations to ordinary differential equations. Later, COCHRAN [2] obtained asymptotic solutions for the von Karman problem while BENTON [3] extended the problem to the transient state. The steady heat transfer above a rotating disk maintained at a constant temperature was examined by MILLSAPS and POHLHAUSEN [4] for a variety of Prandtl numbers while SPARROW and GREGG [5] extended the problem to at any Prandtl number. ATTIA [6] extended the problem discussed in [4,5] to the unsteady state in the presence of an applied uniform magnetic field. The steady flow of a non-Newtonian fluid due to a rotating disk with uniform suction was considered by MiTHAL [7]. Then, ATTIA [8] extended the problem to the transient state with heat transfer. The study of a Newtonian fluid flow in a porous medium is studied by ATTIA [9] in the presence uniform suction and injection.

In the present paper, the time varying laminar flow through a porous medium of an incompressible viscous non-Newtonian fluid due to the uniform rotation of an infinite disk is studied with heat transfer where the Darcy model is assumed [10-12]. The temperature of the disk is maintained at a constant value. The governing nonlinear partial differential equations are integrated numerically using the finite difference approximations The effect of the porosity of the medium and the characteristics of the non-Newtonian fluid on the flow and heat transfer distributions is discussed. 


\section{BASIC EQUATIONS}

The disk is assumed to lie in the plane $\mathrm{z}=0$ and the space $\mathrm{z}>0$ is equipped by a viscous incompressible fluid. The motion is due to the impulsive rotation from rest of an insulated disk of infinite extent about an axis perpendicular to its plane with constant angular speed $\omega$ through a porous medium. The flow in the porous medium deals with the analysis in which the differential equation governing the fluid motion is based on the Darcy's law which accounts for the drag exerted by the porous medium [10-12]. Otherwise the fluid is at rest under pressure $p_{\infty}$. The disk is maintained at a constant temperature $T_{w}$. The non-Newtonian fluid considered in the present paper is that for which the stress tensor $\tau_{j}^{i}$ is related to the rate of strain tensor $e_{j}^{i}$ as [7], $\tau_{j}^{i}=2 \mu e_{j}^{i}+2 \mu_{c} e_{k}^{i} e_{j}^{k}-p \delta_{j}^{i}, e_{j}^{i}=0$,

where $p$ is denoting the pressure, $\mu$ is the coefficient of viscosity and $\mu_{\mathrm{c}}$ is the coefficient of cross viscosity. The governing equations of steady motion are given by

$\frac{\partial u}{\partial r}+\frac{u}{r}+\frac{\partial w}{\partial z}=0$

$\rho\left(\frac{\partial u}{\partial t}+u \frac{\partial u}{\partial r}+w \frac{\partial u}{\partial z}-\frac{v^{2}}{r}\right)+\frac{\mu}{K} u=\frac{\partial \tau_{r}^{r}}{\partial r}+\frac{\partial \tau_{r}^{z}}{\partial z}+\frac{\tau_{r}^{r}-\tau_{\varphi}^{\varphi}}{r}$

$\rho\left(\frac{\partial v}{\partial t}+u \frac{\partial v}{\partial r}+w \frac{\partial v}{\partial z}+\frac{u v}{r}\right)+\frac{\mu}{K} v=\frac{\partial \tau_{\varphi}^{r}}{\partial r}+\frac{\partial \tau_{\varphi}^{z}}{\partial z}+\frac{2 \tau_{\varphi}^{r}}{r}$

$\rho\left(\frac{\partial w}{\partial t}+u \frac{\partial w}{\partial r}+w \frac{\partial w}{\partial z}\right)+\frac{\mu}{K} w=\frac{\partial \tau_{z}^{r}}{\partial r}+\frac{\partial \tau_{z}^{z}}{\partial z}+\frac{\tau_{z}^{r}}{r}$

where $u, v, w$ are velocity components in the directions of increasing $r, \varphi, z$ respectively, $P$ is denoting the pressure, $\mu$ is the coefficient of viscosity, $\rho$ is the density of the fluid, and $K$ is the Darcy permeability [10-12]. Introducing von Karman transformations [1],

$u=r \omega F, v=r \omega G, w=\sqrt{\omega v} H, z=\sqrt{v / \omega} \zeta, p-p_{\infty}=-\rho v \omega P$

where $\zeta$ is a non-dimensional distance measured along the axis of rotation, $F, G, H$ and $P$ are non-dimensional functions of $\zeta$ and $t$, and $v$ is the kinematic viscosity of the fluid, $v=\mu / \rho$ transforms Eqs. (1)-(4) to

$\frac{\partial H}{\partial \zeta}+2 F=0$

$\frac{\partial F}{\partial t}-\frac{\partial^{2} F}{\partial \zeta^{2}}+H \frac{\partial F}{\partial \zeta}+F^{2}-G^{2}+M F+\frac{1}{2} K\left(\left(\frac{\partial F}{\partial \zeta}\right)^{2}+3\left(\frac{\partial G}{\partial \zeta}\right)^{2}+2 F \frac{\partial^{2} F}{\partial \zeta^{2}}\right)=0$

$\frac{\partial G}{\partial t}-\frac{\partial^{2} G}{\partial \zeta^{2}}+H \frac{\partial G}{\partial \zeta}+2 F G+M G-K\left(\frac{\partial F}{\partial \zeta} \frac{\partial G}{\partial \zeta}-F \frac{\partial^{2} G}{\partial \zeta^{2}}\right)=0$ 
$\frac{\partial H}{\partial t}-\frac{\partial^{2} H}{\partial \zeta^{2}}+H \frac{\partial H}{\partial \zeta}+M H+\frac{7}{2} K \frac{\partial H}{\partial \zeta} \frac{\partial^{2} H}{\partial \zeta^{2}}+\frac{\partial P}{\partial \zeta}=0$

where $M=v / K \omega$ is the porosity parameter. The initial and boundary conditions for the velocity problem are given by

$$
\begin{aligned}
& t=0, F=0, G=0, H=0, \\
& \zeta=0, F=0, G=1, H=0, \\
& \zeta \rightarrow \infty, F \rightarrow 0, G \rightarrow 0, P \rightarrow 0,
\end{aligned}
$$

Equation (9a) represents the initial conditions, Eq. (9b) ensures the satisfaction of the no-slip condition of viscous flow applied at the surface of the disk and Eq. (9c) indicates the satisfaction of vanishing of the axial component of velocity. The above system of Eqs. (5)-(7) with the prescribed initial and boundary conditions given by Eq. (9) can be solved to determine the three components of the flow velocity. Equation (8) can be used to solve for the pressure distribution if required.

Due to the difference in temperature between the wall and the ambient fluid, heat transfer takes place. The energy equation without the dissipation terms takes the form [4-5];

$\rho c_{p}\left(\frac{\partial T}{\partial t}+u \frac{\partial T}{\partial r}+w \frac{\partial T}{\partial z}\right)-k\left(\frac{\partial^{2} T}{\partial z^{2}}+\frac{\partial^{2} T}{\partial r^{2}}+\frac{1}{r} \frac{\partial T}{\partial r}\right)=0$

where $T$ is the temperature of the fluid, $c_{p}$ is the specific heat at a constant pressure of the fluid, and $k$ is the thermal conductivity of the fluid.

In terms of the non-dimensional variable

$\theta=\left(T-T_{\infty}\right) /\left(T_{w}-T_{\infty}\right)$

where $T_{w}$ is the temperature at the surface of the disk and $T_{\infty}$ is the temperature of the ambient fluid and using von Karman transformations, Eq. (10) takes the form;

$$
\frac{\partial \theta}{\partial t}-\frac{1}{\operatorname{Pr}} \frac{\partial^{2} \theta}{\partial \zeta^{2}}+H \frac{\partial \theta}{\partial \zeta}=0
$$

where $\operatorname{Pr}$ is the Prandtl number, $\operatorname{Pr}=c_{p} \mu / k$. The initial and boundary conditions in terms of $\theta$ are expressed as

$$
\begin{aligned}
& t=0: \theta=0 \\
& \theta(0)=1, \theta(\infty)=0
\end{aligned}
$$

The heat transfer from the disk surface to the fluid is determined using Fourier's law $Q=-k\left(\frac{d T}{d z}\right)_{w}$

Introducing the transformed variables, the expression for $q$ becomes 
$Q=-k\left(T_{w}-T_{\infty}\right) \sqrt{\frac{\omega}{v}} \frac{\partial \theta(t, 0)}{\partial \zeta}$

By rephrasing the heat transfer results in terms of a Nusselt number defined as, $N_{u}=Q \sqrt{\omega / v} / k\left(T_{w}-T_{\infty}\right)$ the last equation becomes

$N_{u}=-\frac{\partial \theta(t, 0)}{\partial \zeta}$

Numerical solution for the governing nonlinear flow Eqs. (5)-(7) with the conditions given by Eq. (9), using the finite-differences, leads to a numerical oscillation problem resulting from the discontinuity between the initial and boundary conditions (9a) and (9b). The same type of discontinuity happens between the initial and boundary conditions for the energy problem (see Eq. (12)). A suggested solution for this numerical problem is accomplished using suitable coordinate transformations [13] for similar problems. Expressing Eqs. (5)-(7) and (11) in terms of the modified coordinate $\eta=\zeta / 2 \sqrt{t}$, we obtain

$$
\begin{aligned}
& \frac{\partial H}{\partial \eta}+4 \sqrt{t} F=0 \\
& \frac{\partial F}{\partial t}-\frac{\eta}{2 t} \frac{\partial F}{\partial \eta}-\frac{1}{4 t} \frac{\partial^{2} F}{\partial \eta^{2}}+\frac{1}{2 \sqrt{t}} H \frac{\partial F}{\partial \eta}+F^{2}-G^{2}+M F+\frac{K}{8 t}\left(\left(\frac{\partial F}{\partial \eta}\right)^{2}+3\left(\frac{\partial G}{\partial \eta}\right)^{2}+2 F \frac{\partial^{2} F}{\partial \eta^{2}}\right)=0 \\
& \frac{\partial G}{\partial t}-\frac{\eta}{2 t} \frac{\partial G}{\partial \eta}-\frac{1}{4 t} \frac{\partial^{2} G}{\partial \eta^{2}}+\frac{1}{2 \sqrt{t}} H \frac{d G}{d \eta}+2 F G+M G-\frac{K}{4 t}\left(\frac{\partial F}{\partial \eta} \frac{\partial G}{\partial \eta}-F \frac{\partial^{2} G}{\partial \eta^{2}}\right)=0 \\
& \frac{\partial \theta}{\partial t}-\frac{\eta}{2 t} \frac{\partial \theta}{\partial \eta}-\frac{1}{4 t \operatorname{Pr}} \frac{\partial^{2} \theta}{\partial \eta^{2}}+\frac{1}{2 \sqrt{t}} H \frac{\partial \theta}{\partial \eta}=0
\end{aligned}
$$

Equations (13)-(15) represent coupled system of non-linear partial differential equations which are solved numerically under the initial and boundary conditions (9), then Eq. (16) can be obtained using the boundary conditions (12) using the finite difference approximations. A linearization technique is first applied to replace the nonlinear terms at a linear stage, with the corrections incorporated in subsequent iterative steps until convergence is reached. Then the Crank-Nicolson implicit method is used at two successive time levels [13]. An iterative scheme is used to solve the linearized system of difference equations. The solution at a certain time step is chosen as an initial guess for next time step and the iterations are continued till convergence, within a prescribed accuracy. Finally, the resulting block tri-diagonal system is solved using the generalized Thomas-algorithm [13]. Finite difference equations relating the variables are obtained by writing the equations at the mid-point of the computational cell and then replacing the different terms by their second order central difference approximations in the $\eta$-direction. The diffusion terms are replaced by the average of the central differences at two successive timelevels. The computational domain is divided into meshes each of dimension $\Delta t$ and $\Delta \eta$ in time and space respectively. The modified Eqs. (13)-(16) are integrated from $t=0$ to $t=1$. Then, the solution obtained at $t=1$ is used as the initial condition for integrating Eqs. (5)-(7) and (11) from $t=1$ towards the steady state.

The resulting system of equations has to be solved in the infinite domain $0<\eta<\infty$. A finite domain in the $\eta$-direction can be used instead with $\eta$ chosen large enough to ensure that the solutions are not affected by imposing the asymptotic conditions at a finite distance. The independence of the results from the length of the finite domain as well as the grid density was 
ensured and successfully checked by various trial and error numerical experimentations. Computations are carried out for $\eta_{\infty}=10$ and step size $\Delta \eta=0.04$ which are found adequate for the ranges of the parameters studied here. Larger finite distance or smaller step size do not show any significant change in the results. Convergence of the scheme is assumed when all of the variables $F, G, H, \theta, \partial F / \partial \eta, \partial G / \partial \eta$, and $\partial \theta / \partial \eta$ for the last two approximations differs from unity by less than $10^{-6}$ for all values of $\eta$ in $0<\eta<10$ and all $t$.

\section{RESULTS AND DISCUSSION}

Figure 1 presents the evolution of the axial velocity at infinity $H_{f}$ for different values of the non-Newtonian parameter $K$ and the porosity parameter $M$. The figure shows that increasing $K$ decreases the axial flow towards the disk as expected. On the other hand, increasing the parameter $K$ leads to an interesting effect in reversing the direction of the axial velocity for some time which results in a crossover for the charts of $H_{f}$ with time. The time at which the crossover point occurs increases with increasing $K$. Increasing the parameter $M$ decreases the axial flow towards the disk due to its damping effect. It is of interest to see the porosity effect, for non-zero values of $K$, in reversing the direction of the axial flow for all time.

Figure 2 presents the evolution of the Nusselt number $N_{u}$ for various values of the nonNewtonian parameter $K$ and the porosity parameter $M$ and for $\operatorname{Pr}=0.7$. It is depicted that as $K$ or $M$ increases $N_{u}$ decreases. This is due to the fact that increasing $K$ or $M$ resists the axial flow towards the disk and then prevents the fluid at near-ambient temperature to be brought to the neighborhood of the surface of the disk which reduces the heat transfer and, hence, the Nusselt number $N_{u}$.

Figures 3-5 present the influence of the non-Newtonian parameter $K$ on the steady state velocity profiles $G, F$, and $H$, respectively, for the cases $M=0$ and 1 . Figures 3-5 indicate the restraining effect of the porosity of the medium on the flow velocity in the three directions. Increasing the porosity parameter $M$ decreases $G, F$, the magnitude of $H$ and the thickness of the boundary layer. Figure 3 indicates that increasing $K$ increases $G$ for all $\zeta$. Figure 4 indicates that increasing $K$ decreases $F$ for small and moderate $\zeta$. However, for large values of $\zeta$ a crossover point that depends on $K$ appears in $F$ - $\zeta$ charts whereas increasing $K$ more increases $F$. Figure 5 shows that increasing the parameter $K$ increases the restraining effect on the incoming axial flow and consequently decreases the axial velocity towards the disk $H$ for all $\zeta$. A crossover point in $H$ - $\zeta$ charts is also shown in Fig. 5 which indicates that for large values of $K$, the axial flow towards the disk increases with increasing $K$ for small values of $\zeta$. It is of interest to see the effect of the parameter $M$ in the suppression of the crossover in $F$ - $\zeta$ as well as $H$ - $\zeta$ charts as depicted in Figs. 4 and 5. An interesting effect for the parameters $K$ and $M$ appears in Figs. 4 and 5 that for non-zero values of $K$, increasing $M$ reverses the direction of the velocity components $F$ and $H$ for all $\zeta$.

Figure 6 presents of the non-Newtonian parameter $K$ on the steady state temperature profile $\theta$ for the porosity parameter $M=0$ and 1 and for $\operatorname{Pr}=0.7$. Increasing $M$ or the parameter $K$ increases the temperature $\theta$ as a result of the effect of the porosity or the non-Newtonian behavior in preventing the fluid at near-ambient temperature from reaching the surface of the disk. Consequently, increasing $M$ or $K$ increases the temperature as well as the thermal boundary layer thickness. 


\section{CONCLUSION}

In this study the time varying flow of a non-Newtonian fluid induced by a rotating disk with heat transfer in a porous medium was studied. The results indicate the restraining effect of the porosity on the flow velocities and the thickness of the boundary layer. On the other hand, increasing the porosity parameter increases the temperature and thickness of the thermal boundary layer. It is of interest to see the combined effect of the porosity of the medium and the non-Newtonian fluid characteristics on reversing the direction of the radial and the axial components of velocity. Also, it is interesting to see the effect of the porosity parameter in the suppression of the crossover in the radial and axial velocity profiles occur for large values of the non-Newtonian fluid characteristics. In the non-porous case, the non-Newtonian fluid characteristics reverses the direction of the axial flow for some time, but with porosity, the nonNewtonian fluid characteristics reverses the direction of the axial flow for all time.

\section{References:}

[1]VON KARMAN, T., Uber laminare und turbulente reibung, ZAMM, 1 (4) 1921pp. 233-235.

[2]Cochran, W.G., The flow due to a rotating disk, Proc. Cambridge Philos. Soc. 30 (3), pp. 365-375, 1934.

[3]Benton, E.R., On the flow due to a rotating disk, Fluid Mechanics, Vol. 24, No. 4, pp. 781$800,1966$.

[4]Millsaps, K. and PohlHausen, K., Heat transfer by laminar flow from a rotating disk, J. of the Aeronautical Sciences, Vol. 19, pp. 120-126, 1952.

[5]Sparrow, E.M. and GreGG, J.L., Mass transfer, flow, and heat transfer about a rotating disk, ASME J. of Heat Transfer, pp. 294-302, Nov. 1960.

[6]AтTIA, H.A., Unsteady MHD flow near a rotating porous disk with uniform suction or injection, Fluid Dynamics Research 23 (1998) 283-290.

[7]Mithal, K.G., On the effects of uniform high suction on the steady flow of a nonNewtonian liquid due to a rotating disk, Quart. J. Mech. Appl. Math. XIV (1961) 401-410.

[8]AтTiA, H.A., Unsteady flow of non-Newtonian fluid above a rotating disk with heat transfer, International Journal of Heat and Mass Transfer, 46 (2003) 2695-2700.

[9]AтTIA, H.A., On the effectiveness of uniform suction and injection on unsteady rotating disk flow in porous medium with heat transfer, Computational Materials Science, Elsevier Science, Ltd 38 (2006) 240-244.

[10] Joseph, D.D., NiElD, D.A., and PAPANiCOlaOU, G., Nonlinear equation governing flow in a staturated porous media, Water Resources Research 18(4) (1982) 1049-1052.

[11] Ingham, D.B. and PoP, I., Transport phenomena in porous media, Pergamon, Oxford, 2002.

[12]Khaled, A.R.A. and VAFAI, K., The role of porous media in modeling flow and heat transfer in biological tissues, Int. J. Heat Mass Transf. 46 (2003) 4989-5003.

[13] AmES, W.F., Numerical methods in partial differential equations, 2nd ed., Academic Press, New York (1977). 


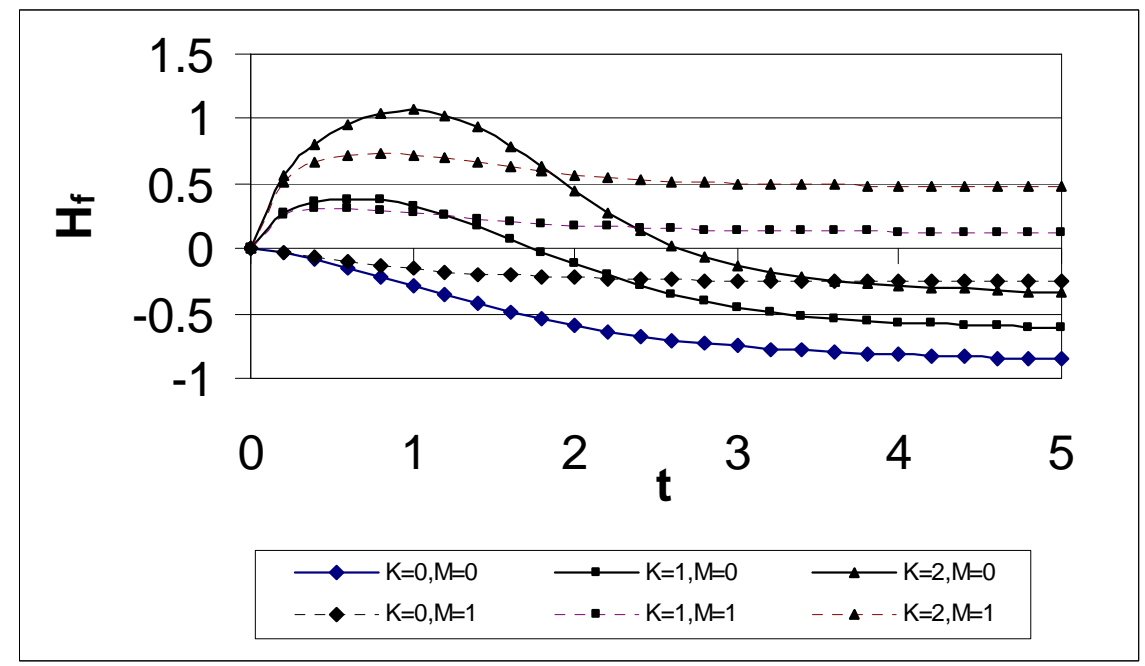

Fig. 1. Time growth of the axial velocity component at infinity $\mathrm{H}_{\mathrm{f}}$ for various values of the parameters $\mathrm{K}$ and $\mathrm{M}$.

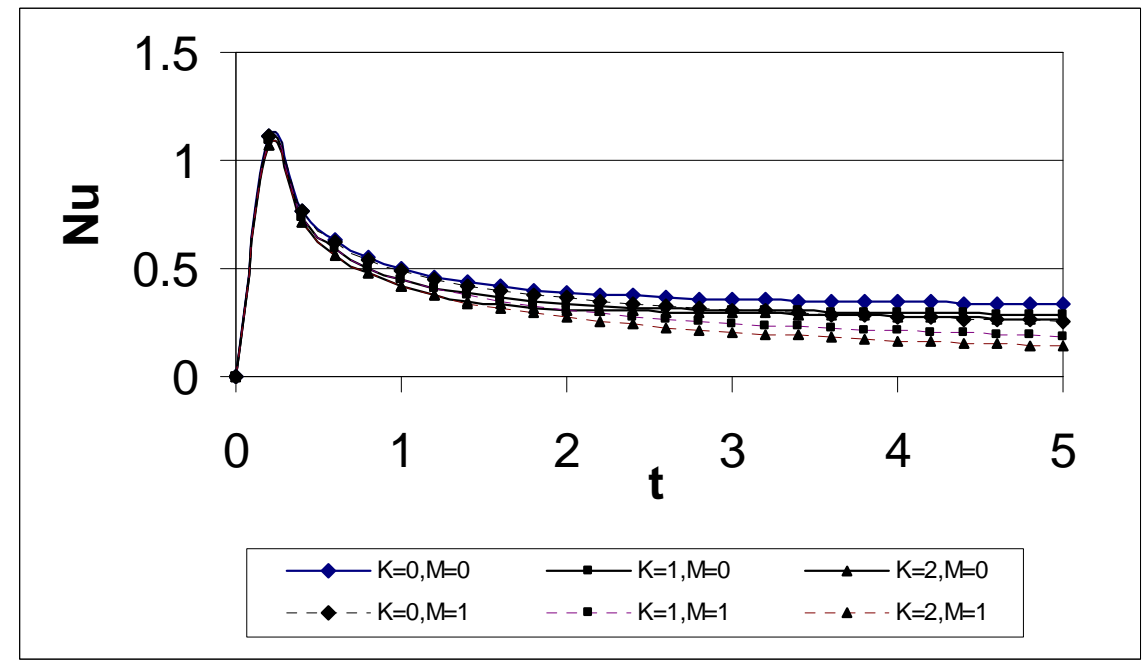

Fig. 2. Time growth of the Nusselt number at the surface of the disk for various values of the parameters $\mathrm{K}$ and $\mathrm{M}$.

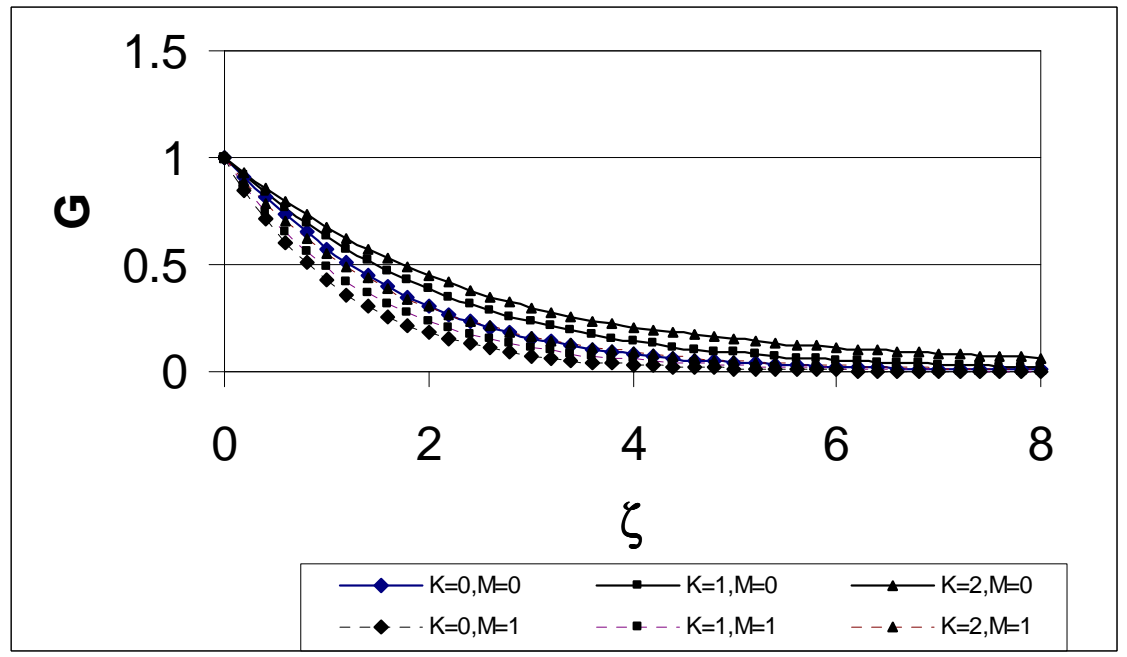

Fig. 3. Effect of the porosity parameter M and the non-Newtonian parameter $\mathrm{K}$ on the profile of $\mathrm{G}$. 


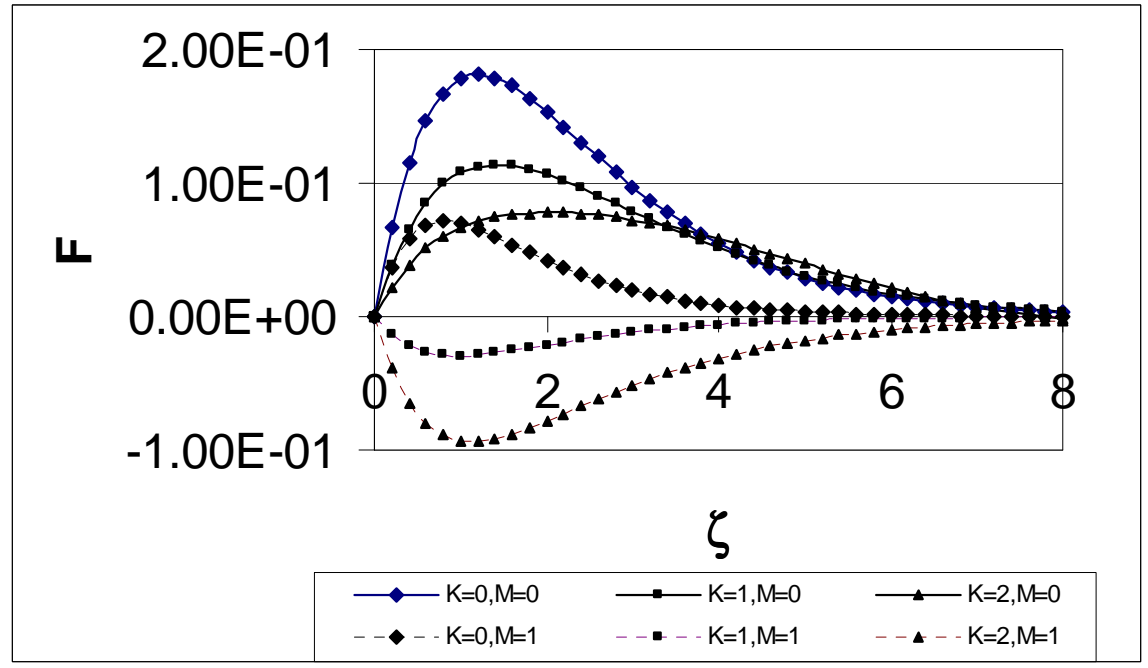

Fig. 4. Effect of the porosity parameter $M$ and the non-Newtonian parameter $K$ on the profile of $F$.

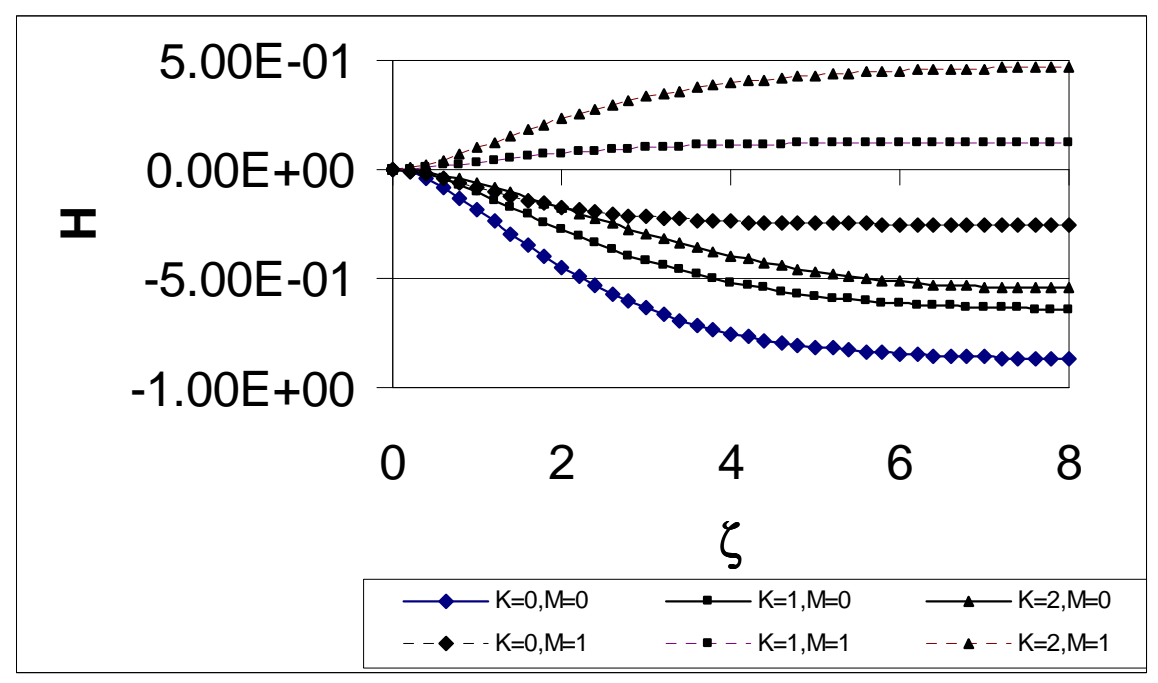

Fig. 5. Effect of the porosity parameter $\mathrm{M}$ and the non-Newtonian parameter $\mathrm{K}$ on the profile of $\mathrm{H}$.

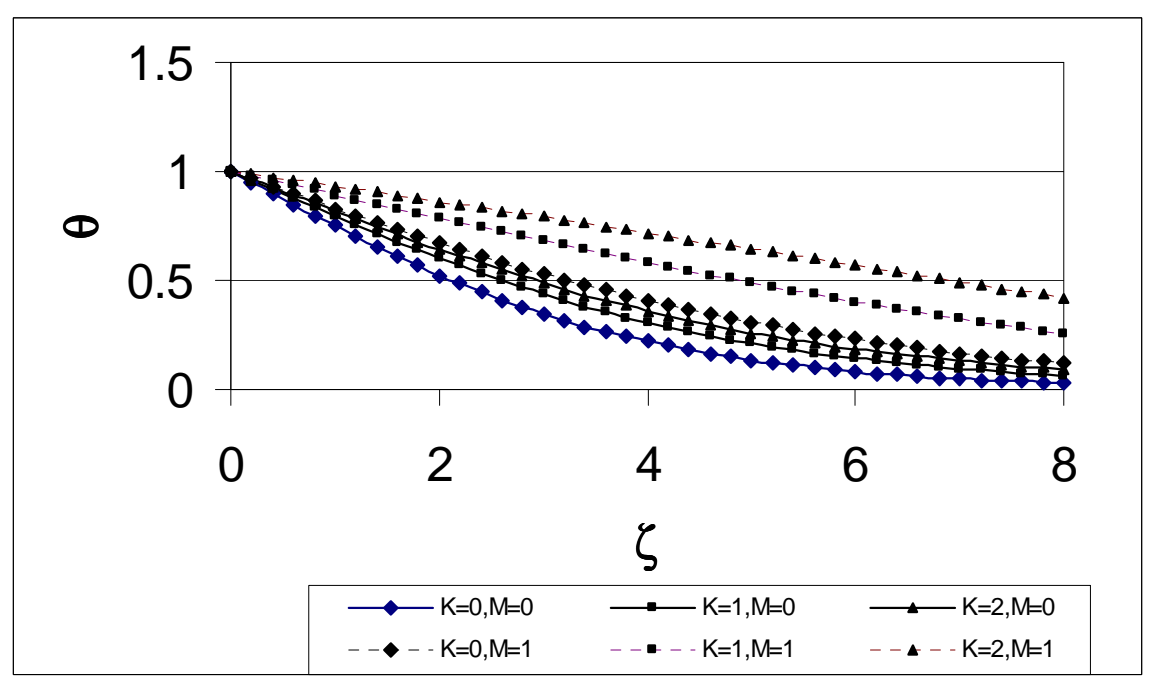

Fig. 6. Effect of the porosity parameter $\mathrm{M}$ and the non-Newtonian parameter $\mathrm{K}$ on the profile of $\theta$. 